\title{
Quand le coupable devient victime
}

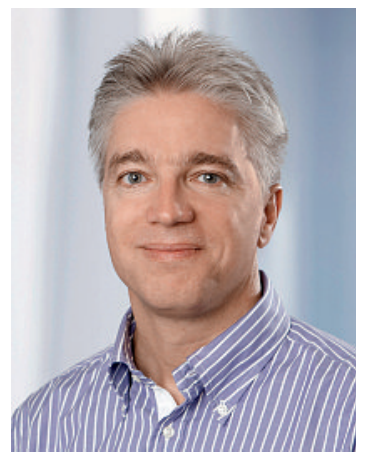

7 h 30 du matin au bloc opératoire, il règne une ambiance studieuse dans toutes les salles. On prépare les patients pour leur intervention, les installe sur les tables d'opération et les endort. Le chirurgien entre dans la salle, contrôle rapidement le travail qui a été fait et se prépare pour son intervention. Il a étudié les dossiers la veille au soir, il est donc mentalement préparé, mais tient à s'assurer par un dernier regard sur le tableau que le premier patient qu'il va opérer est bien celui qui se plaint de douleurs chroniques au coude. Malheureusement, le patient a souhaité une anesthésie générale, il est donc déjà endormi lorsque le chirurgien entre dans la salle. Celui-ci l'aurait encore volontiers salué et répondu aux questions qu'il aurait pu avoir, mais il est vrai que tout a déjà été discuté en détail lors de la consultation. La préparation suit son cours, le patient est

\section{J'ai failli opérer le mauvais côté du coude...}

couvert et le chirurgien, plus par habitude que par nécessité, dessine le tracé principal de l'incision sur la face externe du coude puis s'empare du scalpel. C'est à ce moment qu'il aperçoit par-dessus le rideau médical le visage bienveillant de l'anesthésiste qui semble extrêmement surprise: «C'est intéressant que ce soit la face externe qui soit incisée, car le patient a dit, juste avant de s'endormir, qu'il se réjouissait de voir enfin disparaître les douleurs sur la face interne de son coude!»

Cette histoire, bien réelle, est tirée de mon expérience et je sais que je ne souhaite jamais revivre pareil événement. Heureusement, la collègue se trouvant de l'autre côté du rideau était très attentive; heureusement, il règne entre nous un climat de collégialité et de respect mutuel; heureuse- ment, j'ai remarqué mon erreur à temps avant de nuire à la santé du patient.

Mais ce qui s'est passé en moi à cet instant précis est indescriptible. Comme piqué par une tarentule, j'ai arraché mes vêtements stériles, consulté le dossier du patient et constaté que j'avais effectivement failli opérer le mauvais côté du coude. Cette situation m’a totalement désarçonné;

\section{Bien gérer les erreurs ne va pas de soi.}

je n'ai pas arrêté de me faire des reproches et ai mis en doute mes compétences. Ce n'est que grâce à la grande compréhension de mes collègues et en prenant conscience que je n'avais heureusement causé aucun tort au patient que j'ai $\mathrm{pu}$, après une pause, me ressaisir et continuer ma journée de travail.

Lorsqu'on se retrouve dans une telle situation et si l'on ne peut compter sur le soutien de ses collègues, je peux facilement m'imaginer que même une profession que l'on aime puisse se transformer en cauchemar. L'initiative «Second victim» de la Fondation pour la sécurité des patients mérite donc toute notre attention. Jusqu'alors tabou, ce thème est aujourd'hui abordé de manière systématique dans le but de sensibiliser, mais également de se donner les moyens d'intégrer cette problématique à la formation du personnel de santé. Les premiers interpellés sont les chefs, car ce sont eux qui doivent soutenir leurs collaborateurs lorsque surviennent de tels incidents. Enfin, nous vous recommandons vivement la lecture de l'article à la page 990 du présent numéro du Bulletin des médecins suisses.

Et, au fait, le patient n'a aujourd'hui plus mal au coude et est content du résultat de l'opération. Et quant à moi, j'ai ma foi beaucoup appris de cette expérience.

Dr Daniel Herren MHA, Membre du Comité central de la FMH et responsable du domaine $D D Q$ 